\title{
Ultrasound-guided cooled radiofrequency ablation of the genicular nerves: a technique paper
}

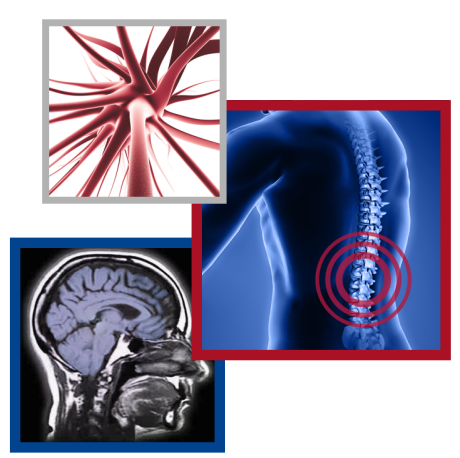

\author{
Dustin Lash*,1, Earl Frantz ${ }^{2}$ \& Mark FB Hurdle ${ }^{3}$ \\ ${ }^{1}$ Southern Bone \& Joint Specialists P.C., Dothan, AL 36301, USA \\ ${ }^{2}$ Carolinas Center for Surgery, Morehead City, NC 28557, USA \\ ${ }^{3}$ Mayo Clinic, Jacksonville, FL 32224, USA \\ *Author for correspondence: sportsdoc@southernbone.com
}

\begin{abstract}
Aim: Cooled radiofrequency ablation (CRFA) has demonstrated efficacy in the management of knee pain caused by osteoarthritis. A typical procedure involves fluoroscopic placement of internally cooled radiofrequency probes to ensure the probes are located near target genicular nerves. Patients $\&$ methods: A new technique was developed to perform CRFA using ultrasound (US) guidance. Patient outcomes were reported using a telephone survey. Results: Ablation procedures were successfully performed using US guidance. A total of 51 patients were retrospectively identified for telephone screening. A total of 22 patients completed the telephone questionnaire. There were no safety concerns identified and the majority of patients reported satisfactory outcomes. Conclusion: Patient outcomes demonstrate that US-guided CRFA procedures provide pain relief and functional improvement. Further studies are needed to compare the efficacy of US-guided CRFA to fluoroscopy-guided CRFA procedures.
\end{abstract}

First draft submitted: 23 December 2019; Accepted for publication: 17 February 2020; Published online: 4 March 2020

Keywords: cooled radiofrequency ablation $\bullet$ knee osteoarthritis $\bullet$ nonoperative pain management $\bullet$ opioid free

Knee pain as a result of symptomatic osteoarthritis (OA) is a common condition affecting nearly 14 million individuals in the USA [1]. Total knee replacement (TKR) is generally considered the definitive treatment for knee OA. As durability of TKR is approximately 20 years, many surgeons prefer to delay surgery, so that patients will only undergo one TKR. Furthermore, many patients are not candidates for surgery due to a number of co-morbidities or personal preference to not undergo surgery [2]. Minimally invasive procedures that can help manage pain are an important part of the treatment paradigm for patients experience pain related to knee OA.

Radiofrequency ablation (RFA) is the targeted thermal degradation of sensory nerves with the intent to relieve pain. As radiofrequency energy is emitted from a probe tip, ionic heating will heat the surrounding tissues to temperatures of $80^{\circ} \mathrm{C}$. Pain is believed to be attenuated while the nerve structure heals. Extended periods of ionic heating with standard radiofrequency probes creates charring at the tissue-tip interface, preventing further delivery of energy. As such, lesions created by standard RFA are elliptical in shape. Standard RFA has shown a durability of 3-6 months when managing knee OA pain [3,4].

Internally cooled radiofrequency probes are able to overcome the shortcomings inherent to standard radiofrequency probes. In cooled radiofrequency probes, circulated water carries heat away from the tissue-tip interface, mitigating charring and enabling the delivery of more energy. While the probe temperature is set at $60^{\circ} \mathrm{C}$, tissues in the surrounding areas reach $80^{\circ} \mathrm{C}$. Lesions created by cooled radiofrequency ablation (CRFA) are larger in size and nerve structure is more extensively damaged [5,6]. Larger lesion size has been hypothesized to improve treatment success, as a larger lesion may help overcome nerve anatomical variability. CRFA treatments have been shown to reduce pain by over $50 \%$ in roughly $65 \%$ of patients 6 months post-procedure $[7,8]$. Furthermore, studies have shown that this pain relief can often extend to 12 months [9].

Proper probe placement is crucial during CRFA procedures as to ensure the highest chance of therapeutic success. The traditional visualization technique for probe placement is fluoroscopy. A new alternative visualization technique has been developed utilizing ultrasound (US) guidance to assist in the placement of cooled radiofrequency probes.

Future $\because$ Medicine 
There are several advantages to utilizing US including zero exposure to radiation, more precise needle placement and soft tissue visualization. Additionally, US equipment is more widely available than fluoroscopy, potentially creating better access for patients seeking this treatment.

\section{Patients \& methods}

\section{Development of US-guided CRFA technique}

The technique presented in this manuscript was first developed by using cadaveric models. The authors convened to a facility where fluoroscopy equipment, US equipment and three cadavers were present. Needles were first placed via US based on bony landmarks frequently used in fluoroscopic techniques, which are, in turn, informed by robust anatomical studies $[10,11]$. To confirm proper needle placement, fluoroscopic images were taken to ensure needles were placed in appropriate location. One shortcoming of US on cadaver models is that, while it is possible visualize bony landmarks and nerve structures, blood flow is not visible. On live models, blood flow serves as a confirmation, as the location nerve structures relative to arteries is well documented in the knee.

\section{Data collection}

The data collection portion of this manuscript was an Institutional Review Board (IRB)-approved investigatorinitiated retrospective study. Patients were retrospectively identified and contacted via telephone using a standardized questionnaire with questions pertaining to pain following US-guided CRFA. Patients were consented during this telephone call. Inclusion criteria consisted of subjects who received US-guided CRFA at Carolinas Center for Surgery from October 2017 to October 2018. Those receiving bilateral procedures were not excluded from analysis. As the goal of this was to provide a general overview of patient outcomes following procedure, inclusion criteria was purposefully broad.

\section{US-guided CRFA technique overview}

Utilizing a linear or curvilinear US transducer, the target nerves in each patient were identified using US guidance. The targets for this procedure were the superior medial, inferior medial, superior lateral genicular nerves and the suprapatellar genicular nerve. Robust anatomical studies have identified the key sensory nerves innervating the knee joint, which are believed to be responsible for relaying signals related to chronic knee pain $[10,11]$. Once the nerves or their corresponding landmarks were identified, a 17-gauge introducer was advanced to a location near the nerve under US visualization. The technique used to identify each nerve and place the introducer is detailed below.

\section{Patient positioning \& preparation}

- Place the patient in the supine position with the ipsilateral knee elevated using towels (knee flexed $25-30^{\circ}$ );

- Sterile prep and drape using strict aseptic technique.

\section{Superior medial geniculate nerve}

- Align US transducer in a coronal orientation over the medial joint line and move cephalad to the metaphyseal/diaphyseal junction;

- Identify the genicular artery nerve and artery (long axis), which are generally located near the periosteum. If the neurovascular bundle is poorly visualized, use the metaphyseal/diaphyseal junction as a bony landmark for introducer placement;

- Mark the skin at the midpoint of the US transducer corresponding to the genicular artery;

- Turn the US transducer to an axial orientation to view the genicular artery/nerve in the short axis. If visualization is not possible, confirm $50 \%$ depth of femur;

- Anesthetize skin and soft tissues with 1\% lidocaine;

- Advance the introducer anterior to posterior using an in-plane approach to the genicular nerve/artery or 50\% depth of the femur;

- Turn the US transducer to a coronal orientation and verify the introducer tip is near the genicular artery/nerve or the metaphyseal/diaphyseal junction (Figure 1);

- Remove stylet, insert the probe and stimulate the geniculate nerve branch at $2 \mathrm{~Hz}$ up to $1-2 \mathrm{~V}$ searching for any lower extremity motor activity. If no motor response is observed, then and inject local anesthetic into the introducer;

- Perform CRFA with lesion settings at $60^{\circ} \mathrm{C}\left(80-90^{\circ}\right.$ adjacent tissue temperature) for 2:30 min. 


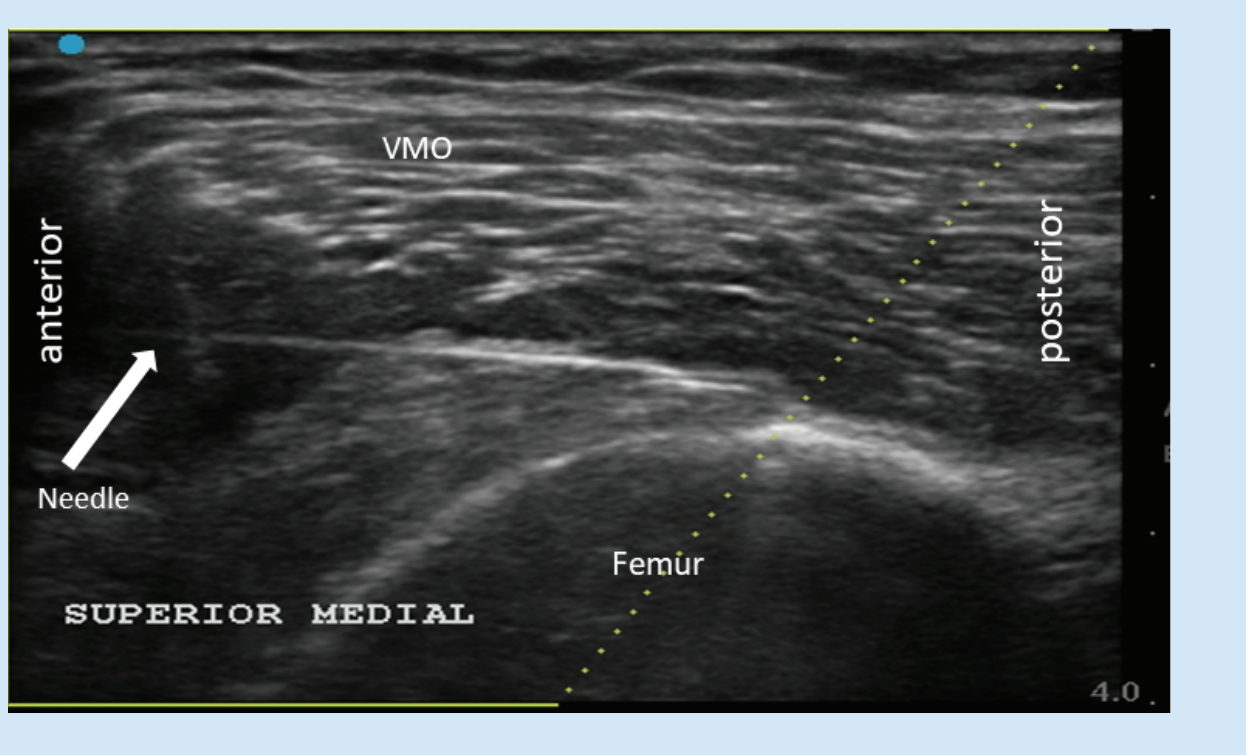

Figure 1. Superior medial needle placement.

VMO: Vastus medialis obliquus.

Inferior medial geniculate nerve

- Align US transducer in a coronal orientation over the medial joint line and move caudal to the metaphyseal/diaphyseal junction;

- Identify the genicular artery nerve and artery (long axis) that are generally located near the periosteum. If the neurovascular bundle is poorly visualized, use the metaphyseal/diaphyseal junction as a bony landmark for introducer placement;

- Mark the skin at midpoint of US transducer corresponding to the genicular artery;

- Turn the US transducer to an axial orientation to view the genicular artery/nerve in short axis. If visualization is not possible, confirm $50 \%$ depth of tibia;

- Anesthetize skin and soft tissues with $1 \%$ lidocaine;

- Advance the introducer anterior to posterior using an in-plane approach to the genicular nerve/artery or $50 \%$ depth of the tibia;

- Turn the US transducer into a coronal orientation and verify the introducer tip is near the genicular artery/nerve or the metaphyseal/diaphyseal junction (Figure 2);

- Remove stylet, insert probe and stimulate geniculate nerve branch at $2 \mathrm{~Hz}$ up to $1-2 \mathrm{~V}$ searching for any lower extremity motor activity. If no motor response is observed, then and inject local anesthetic into introducer;

- Perform CRFA with lesion settings at $60^{\circ} \mathrm{C}\left(80-90^{\circ}\right.$ adjacent tissue temperature) for 2:30 min.

\section{Superior lateral genicular nerve}

- Align US transducer in a coronal orientation over the lateral joint line and move cephalad to the metaphyseal/diaphyseal junction;

- Identify the genicular artery nerve and artery (long axis), located near the periosteum. If the neurovascular bundle is poorly visualized, use the metaphyseal/diaphyseal junction as a bony landmark for introducer placement;

- Mark the skin at midpoint of US transducer corresponding to the genicular artery;

- Turn the US transducer to an axial orientation to view the genicular artery/nerve in short axis. If visualization is not possible, confirm $50 \%$ depth of femur;

- Anesthetize skin and soft tissues with $1 \%$ lidocaine;

- Advance the introducer anterior to posterior using an in-plane approach to the genicular nerve/artery or 50\% depth of the femur;

- Turn the US transducer into a coronal orientation and verify the introducer tip is near the genicular artery/nerve or the metaphyseal/diaphyseal junction (Figure 3); 


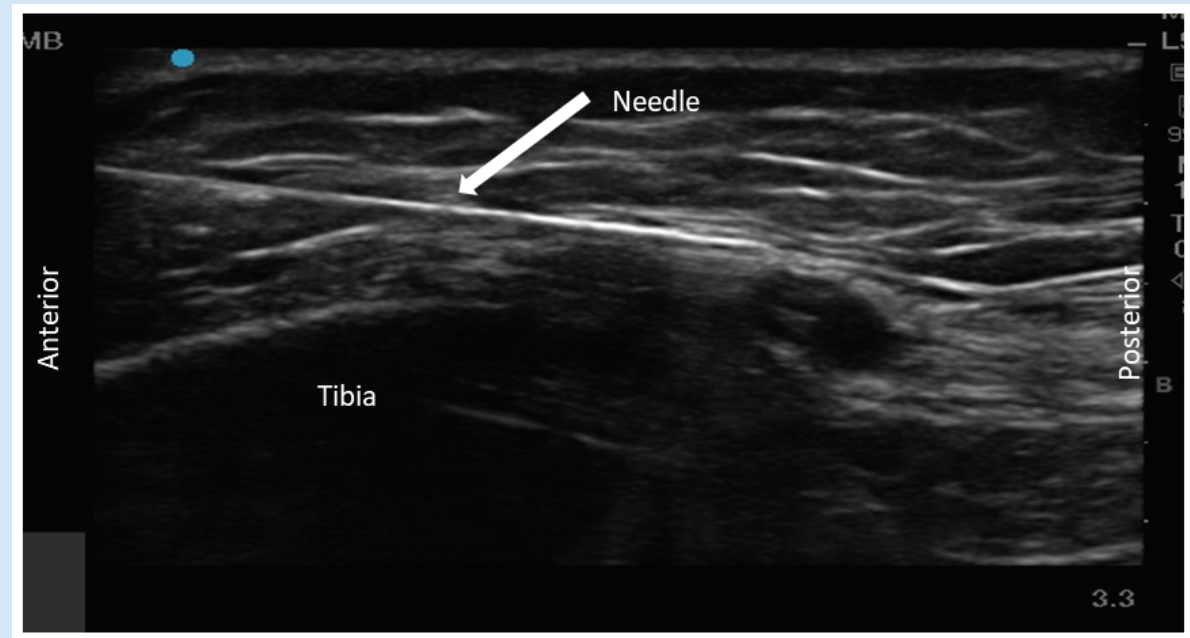

Figure 2. Inferior medial needle placement.

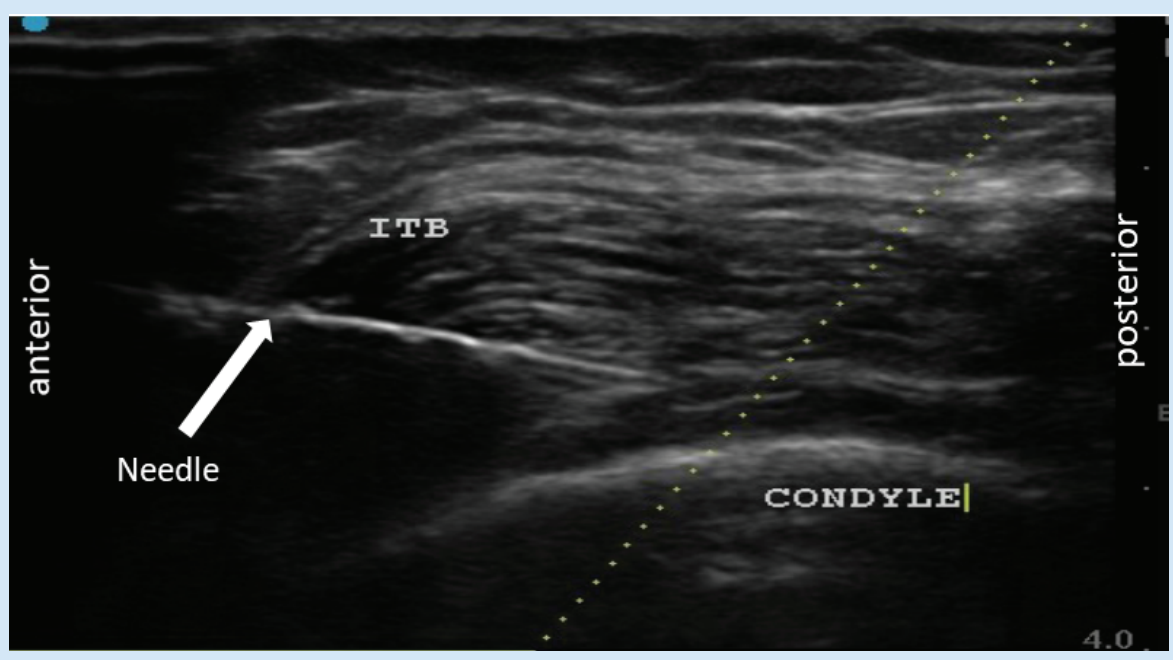

Figure 3. Superior lateral needle placement.

ITB: Iliotibial band.

- Remove stylet, insert probe and stimulate geniculate nerve branch at $2 \mathrm{~Hz}$ up to $1-2 \mathrm{~V}$ searching for any lower extremity motor activity. If no motor response is observed, then and inject local anesthetic into introducer;

- Perform CRFA with lesion settings at $60^{\circ} \mathrm{C}\left(80-90^{\circ}\right.$ adjacent tissue temperature $)$ for 2:30 min.

\section{Suprapatellar genicular nerve}

- Align midpoint of US transducer in sagittal orientation approximately $5 \mathrm{~cm}$ proximal to the superior pole of the patella to visualize the quadriceps tendon, prefemoral fat pad and femur;

- If an effusion is present, move the transducer to its proximal border;

- Turn the US transducer $90^{\circ}$ to visualize the femur and quadriceps tendon in short axis;

- Measure down to the depth of the fat pad just superficial to the femur;

- Anesthetize skin and soft tissues with 1\% lidocaine; 


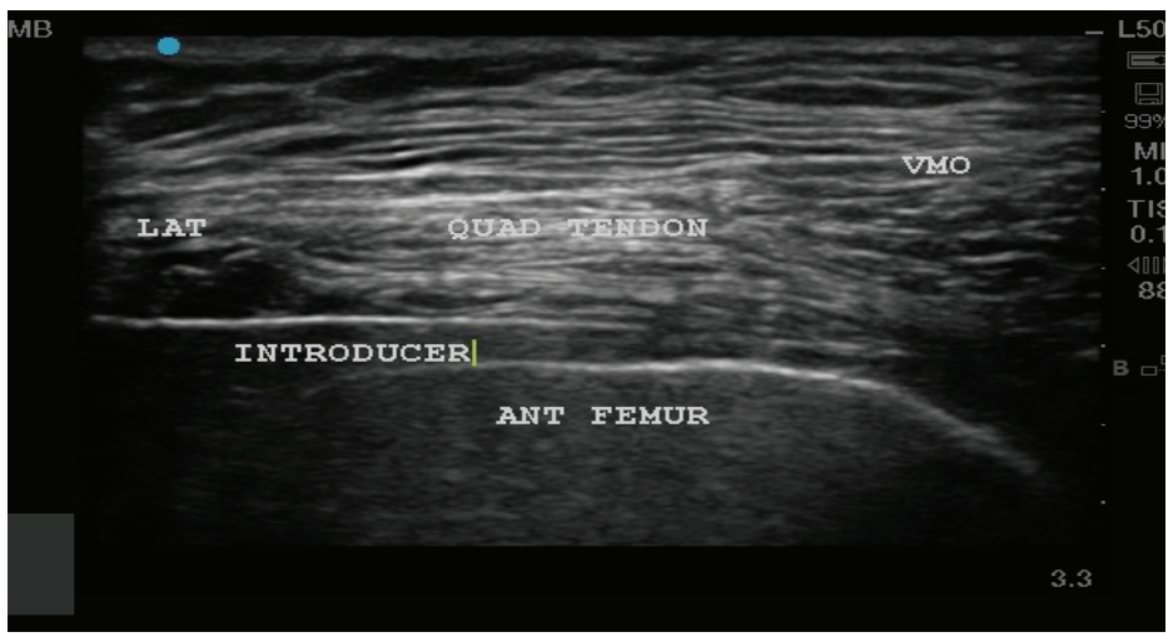

Figure 4. Suprapatellar introducer placement.

ANT: Anterior; LAT: Lateral; VMO: Vastus medialis obliquus.

- Insert the introducer in plane to midline of the femur/quadricep tendon just superficial to the periosteum (Figure 4);

- Remove stylet, insert probe and stimulate geniculate nerve branch at $2 \mathrm{~Hz}$ up to $1-2 \mathrm{~V}$ searching for any lower extremity motor activity. If no motor response is observed, then and inject local anesthetic into introducer;

- Perform CRFA with lesion settings at $60^{\circ} \mathrm{C}\left(80-90^{\circ}\right.$ adjacent tissue temperature) for 2:30 min.

\section{Fluoroscopic confirmation of technique}

When using fluoroscopic placement, needles are advanced to the bony end points on the superolateral portion of the femoral condyle, the superomedial portion of the femoral condyle, the inferomedial portion of the tibial and at the midline of the femur, about 2-cm cephalad of the upper patellar border. Lateral $\mathrm{x}$-ray views were created to confirm appropriate location at $50 \%$ depth of the femur and tibia prior to lesioning. US guidance facilitated successful placement of the radiofrequency probes in close proximity to the genicular nerves or the fluoroscopic landmarks of the genicular nerve pathway in these patients. Introducer placement was verified by fluoroscopic imaging to verify similar needle placement to original fluoroscopic placement technique (Figures 5 \& 6).

\section{Results}

\section{Patient outcomes}

All patients underwent US-guided CRFA on the four identified genicular nerves. Follow-up with patients consisted of a telephone questionnaire related to pain and function (Table 1).

A total of 51 patients were retrospectively identified during the specified treatment period. Attempts were made to contact each of these subjects. Data were collected from 22 patients, representing 33 knee procedures $(11$ bilateral and 11 unilateral). Demographics of this population are detailed in Table 2. The mean time between procedure and data collection was 306 days (min 162 days, max 519 days).

Responses to the telephone questionnaire for bilateral and unilateral are reported in Tables $3 \& 4$, respectively. Overall, $82 \%$ of patients contacted reported improvements in pain ranging from 50 to $100 \%$ improvement after receiving US-guided CRFA. Seventy-nine percent of patients reported tolerable functional pain scores after receiving US guided CRFA. Seventy percent of patients reported that their pain does not prevent activity.

\section{Adverse events}

Adverse events were recorded in all subjects receiving US-guided CRFA. Two subjects passed away between treatment and follow-up call (heart attack, multiple myeloma). One subject broke their ankle as a result of falling from a curb. One subject received an intra-articular steroid injection for effusion and tightness (not pain) in treated 


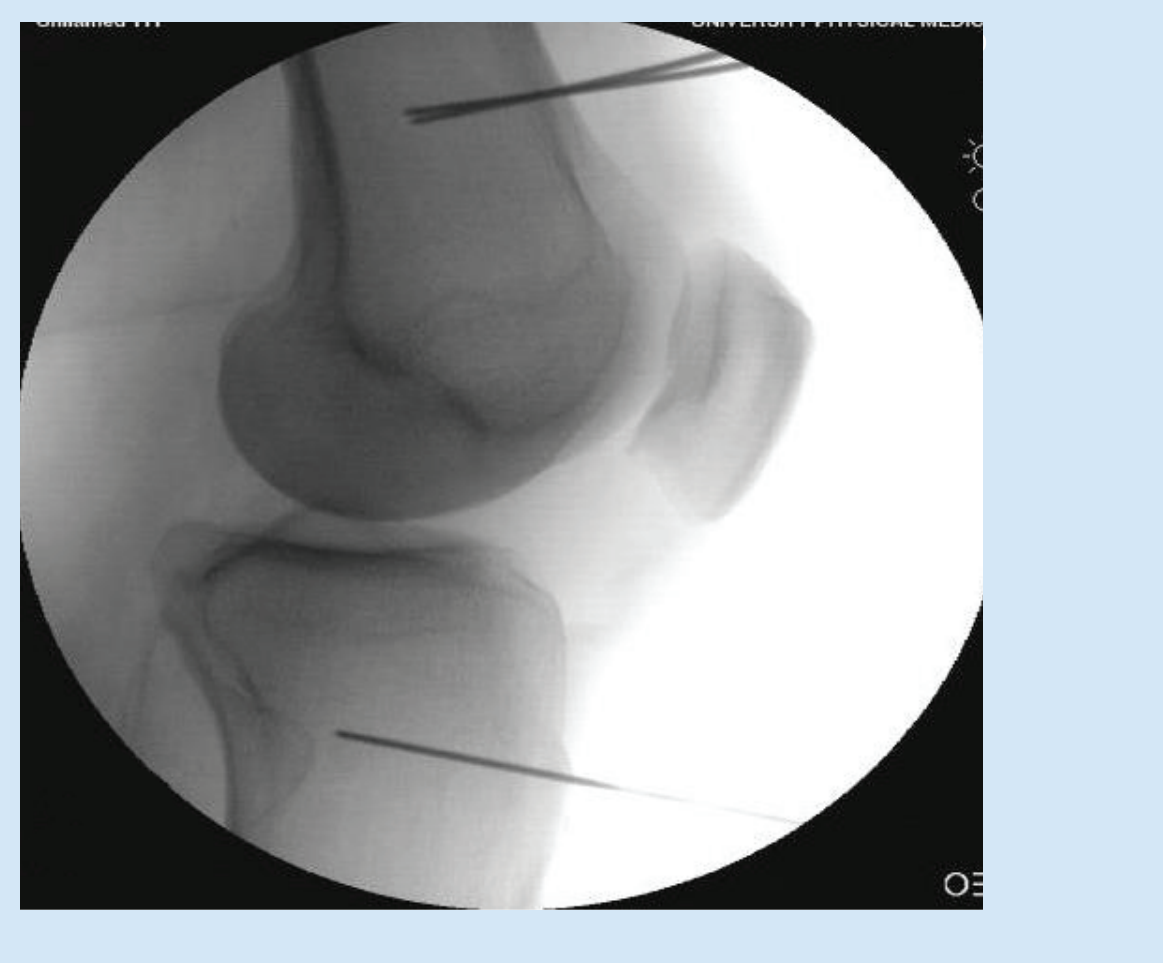

Figure 5. Lateral view: fluoroscopic verification of probe placement for superior medial and lateral genicular nerves and medial distal genicular nerve.

\section{Table 1. Telephone questionnaire.}

For each knee, please rate the following on a scale from 0 to 10 , where 0 is no pain and 10 is the worst possible pain. CURRENT: What is your CURRENT pain level

REST: What is your BEST pain level with rest?

REST: What is your WORST pain level with rest?

REST: What is your AVERAGE pain level with rest?

ACTIVITY: What is your BEST pain level with activity?

ACTIVITY: What is your WORST pain level with activity?

ACTIVITY: What is your AVERAGE pain level with activity?

Since your ultrasound-guided cooled radiofrequency ablation procedure, what percent improvement have you achieved?

Since your ultrasound-guided cooled radiofrequency ablation procedure, how would you describe your function or ability to carry out activities? Choose one answer:

$0=$ no pain

$1=$ tolerable and pain does not prevent any activity

$2=$ tolerable and pain prevents some activities

$3=$ intolerable and pain does not prevent use of telephone, TV viewing or reading

$4=$ intolerable and pain does prevent use of telephone, TV viewing or reading

$5=$ intolerable and pain prevents verbal communication

\section{Table 2. Subject demographics.}

\begin{tabular}{|c|c|c|c|c|c|}
\hline \multicolumn{2}{|c|}{ Combined gender } & \multicolumn{2}{|c|}{ Combined age } & \multicolumn{2}{|c|}{ Days from procedure to follow-up call } \\
\hline Female & 16 & Avg & 72 & Avg & 309 \\
\hline Male & 6 & Min & 53 & Min & 162 \\
\hline \multirow[t]{2}{*}{ Total } & 22 & Max & 92 & Max & 519 \\
\hline & & Median & 73 & Median & 279 \\
\hline
\end{tabular}




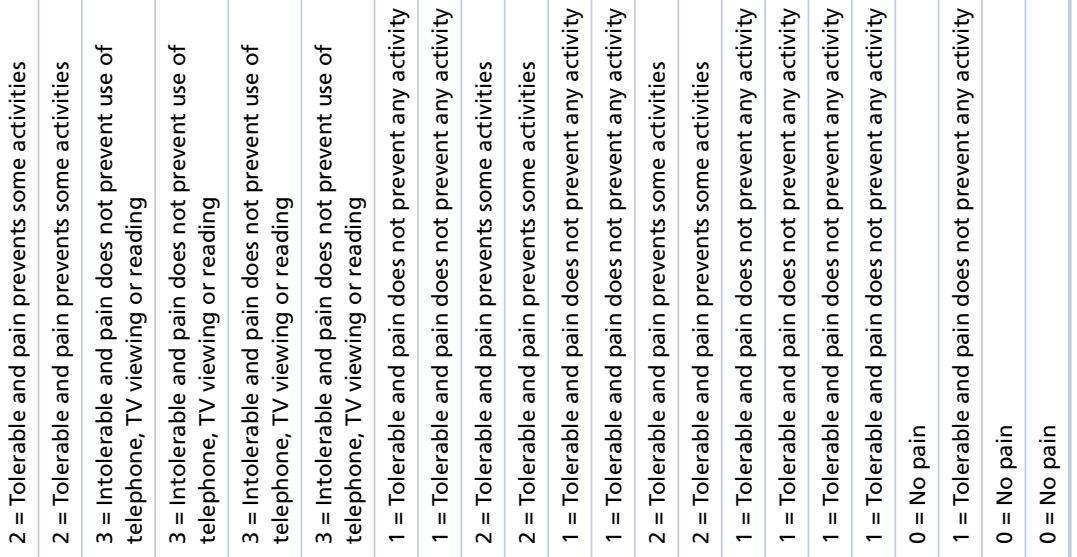

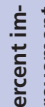

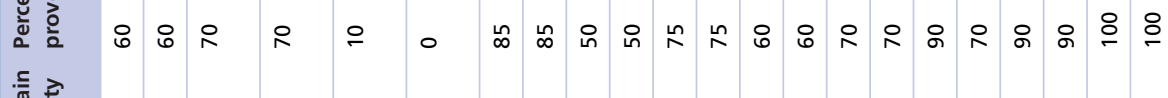

跤

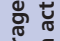

这

든

辛

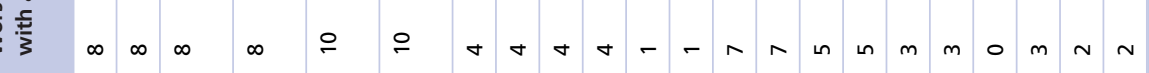

站

㟧

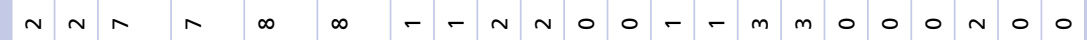

要

姿蒙

등

蒿 


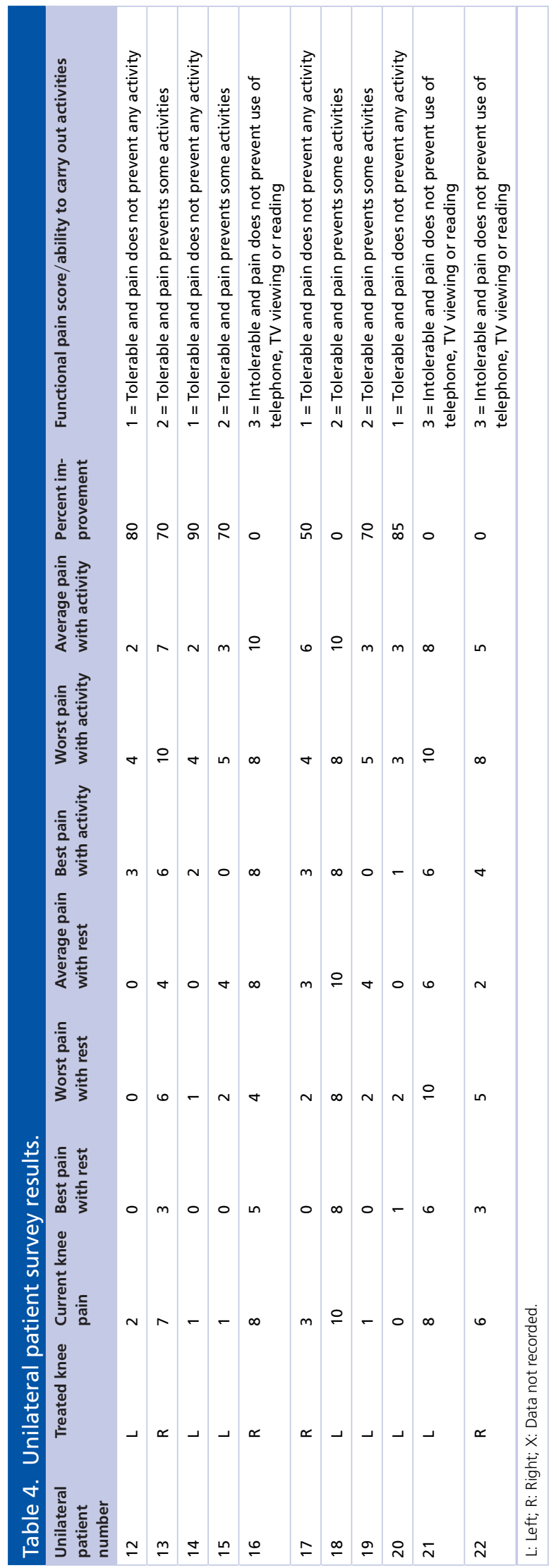




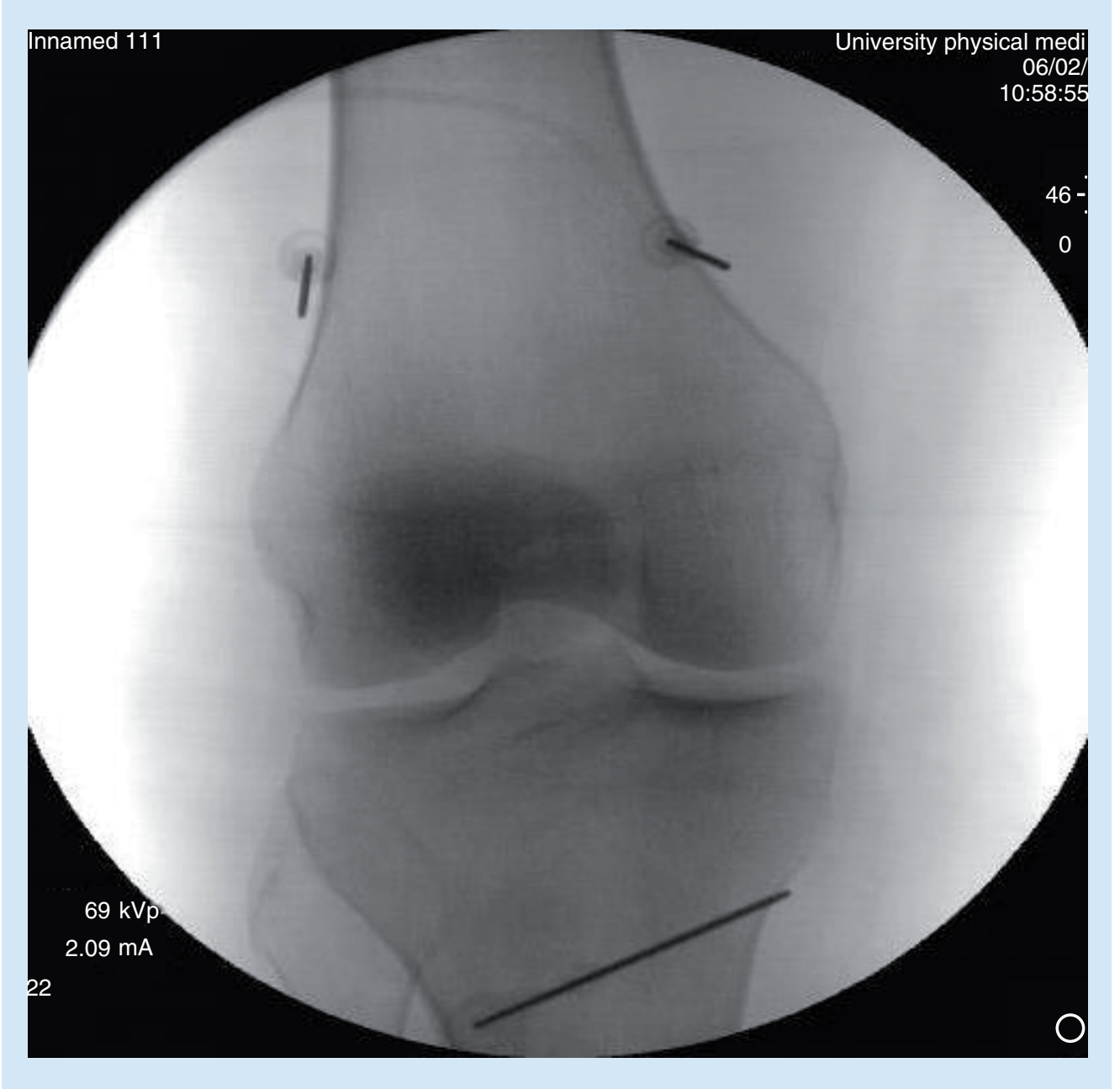

Figure 6. Anterior/posterior view: fluoroscopic verification of probe placement for superior medial and lateral genicular nerves and medial distal genicular nerve.

knee 7 weeks post-treatment. There were three instances of Pes Anserine Bursitis requiring steroid injections, which were not deemed related to the procedure.

\section{Discussion}

The development of new techniques for performing CRFA represents a valuable tool in the management of knee OA pain. The majority of CRFA procedures done under fluoroscopic guidance are conducted in a hospital setting. By introducing this US technique, this validated procedure is posited to become available to a wider network of both medical professionals and patients alike. Previous studies have also demonstrated the high variability of the genicular nerve course around the knee [10,12]. The utilization of US to guide placement of introducers theoretically allows for optimal placement and direct visualization of genicular nerves, with the added benefit of eliminating the risks associated with radiation exposure. It is well established that radiation exposure can vary based on what procedure is being performed, but research showed the average adult effective dose for interventional fluoroscopy was between 5 and $70 \mathrm{mSv}$ [13]. While medical professionals agree that the benefits of fluoroscopy imaging outweigh the risks associated with radiation exposure, in 2010 the US FDA released an initiative to reduce unnecessary radiation exposure from medical imaging, which focused on the promotion of the safe use of medical imaging devices supporting informed clinical decision-making and increasing patient awareness [14].

Prior literature has discussed the development of US techniques for the administration of nerve block when managing knee OA pain [15]. While this technique and the technique presented within this manuscript share 
similar methodology, the authors believe that the technique presented within this manuscript adds to the body of knowledge, as the methods detailed in this manuscript help further the therapeutic utilization of CRFA procedures. Additionally, this manuscript is accompanied by a training video, which will help practicing medical professionals understand this technique at more detailed level.

Previous clinical literature has confirmed the safety and efficacy of RFA when managing chronic knee pain caused by OA [3,4,7-9]. Previous studies have shown the CRFA is superior to intra-articular steroid injections at the 6-month timepoint [7]. Davis et al. demonstrated that the majority of subjects receiving CRFA experienced a more than 50\% reduction in pain lasting 12 months [9]. More recent trials have confirmed 6-month durability of CRFA and superiority over hyaluronic acid injections [8]. In these trials, CRFA is conducted under fluoroscopic guidance. While the patient-reported end points in this manuscript are not the same used in recent CRFA clinical trials (including Visual Analog Scale pain scores, Numeric Rating Scales of pain, Western Ontario and McMaster Universities Osteoarthritis Index), the authors are encouraged that the initial data from these patients suggest this technique may provide similar outcomes. Additional prospective studies using clinically validated outcomes should be conducted utilizing US for cooled radiofrequency probe placement to assure that relief of knee pain is achieved and is as effective as the techniques described with conventional fluoroscopy.

Limitations of this study include the use of nonclinically validated end points, the lack of statistical power and significance, the lack of prior medical history of study subjects and broad inclusion criteria. Future, prospective, randomized clinical trials should be designed to incorporate these factors to provide a more robust comparison of this technique with fluoroscopic guidance.

\section{Conclusion}

CRFA is a safe and effective treatment when managing chronic knee pain caused by OA. While the procedure typically involves fluoroscopy-guided probe placement, a new US technique has been developed that has the potential for several benefits, including the lack of radiation exposure and ubiquitous equipment availability. Preliminary patient outcomes following US-guided CRFA procedures, as measured by retrospective telephone surveys, indicate that procedures provide pain relief and functional improvement. Further clinical studies are necessary to compare efficacy of UG-guided CRFA to fluoroscopy-guided CRFA procedures.

\section{Summary points}

- Cooled radiofrequency ablation is a safe and effective treatment for pain caused by knee osteoarthritis.

- Radiofrequency probe placement is informed by robust anatomical studies of the nerves innervating the knee joint capsule.

- Fluoroscopic guidance is used to place radiofrequency probes but has several drawbacks.

- Ultrasound-guided probe placement offers several advantages to fluoroscopy.

- Future studies are needed to determine any difference in clinical outcomes between fluoroscopy- and ultrasound-guided cooled radiofrequency ablation.

Financial \& competing interests disclosure

This study was funded in part by Avanos Medical, Inc. D Lash, E Frantz and MFB Hurdle have consulted for Avanos Medical, Inc. The authors have no other relevant affiliations or financial involvement with any organization or entity with a financial interest in or financial conflict with the subject matter or materials discussed in the manuscript apart from those disclosed.

Medical writing assistance was provided by Eric Moorhead (Avanos Medical, Inc.).

Ethical conduct of research

This was an IRB approved investigator initiated retrospective study. Patients were retrospectively identified and contacted via telephone and surveyed for pain-related patient using a telephone recruitment screening script.

\section{References}

Papers of special note have been highlighted as: $\bullet$ of interest

1. Deshpande BR, Katz JN, Solomon DH et al. Number of persons with symptomatic knee osteoarthritis in the US: impact of race and ethnicity, age, sex, and obesity: symptomatic knee OA in the US. Arthritis Care Res. 68(12), 1743-1750 (2016).

2. Kamaruzaman H, Kinghorn P, Oppong R. Cost-effectiveness of surgical interventions for the management of osteoarthritis: a systematic review of the literature. BMC Musculoskelet. Disord. 18(1), 183 (2017). 
3. Choi WJ, Hwang SJ, Song JG et al. Radiofrequency treatment relieves chronic knee osteoarthritis pain: a double-blind randomized controlled trial. Pain 152(3), 481-487 (2011).

4. Sarı S, Aydın ON, Turan Y, Özlülerden P, Efe U, Omürlü I. Which one is more effective for the clinical treatment of chronic pain in knee osteoarthritis: radiofrequency neurotomy of the genicular nerves or intra-articular injection? Int. J. Rheum. Dis. 21(10), 1772-1778 (2018).

5. Ball RD. The science of conventional and water-cooled monopolar lumbar radiofrequency rhizotomy: an electrical engineering point of view. Pain Physician 17, E175-E211 (2014).

- This is an overview of the technology behind internally cooled radiofrequency probes.

6. Zachariah C, Mayeux J, Alas G et al. Cooled radiofrequency ablation produces enhanced thermal lesions in-vivo. ORS Annual Meeting. Austin, TX, USA (2019). https://www.morressier.com/article/1836-cooled-radiofrequency-ablation-produces-enhanced-thermallesions-vivo/5c0a5c98f0ad58000b08a394?

7. Davis T, Loudermilk E, DePalma $\mathrm{M}$ et al. Prospective, multicenter, randomized, crossover clinical trial comparing the safety and effectiveness of cooled radiofrequency ablation with corticosteroid injection in the management of knee pain from osteoarthritis. Reg. Anesth. Pain. Med. 43(1), 84-91 (2018).

- This is a clinical trial demonstrating the efficacy of cooled radiofrequency ablation (CRFA) compared with steroid injections in the management of knee osteoarthritis (OA) pain. The majority of subjects receiving CRFA receive pain relief lasting at least 6 months.

8. Chen AF, Khalouf F, Zora K et al. Cooled radiofrequency ablation demonstrates greater efficacy and equivalent safety compared to a single injection of hyaluronic acid for chronic knee pain management: a multi-center, randomized, clinical trial. J. Bone Joint Surg. (2019) (In Press).

- This is a clinical trial demonstrating the efficacy of CRFA compared with hyaluronic acid injections in the management of knee OA pain. The majority of subjects receiving CRFA receive pain relief lasting at least 6 months.

9. Davis T, Loudermilk E, DePalma $\mathrm{M}$ et al. Twelve-month analgesia and rescue, by cooled radiofrequency ablation treatment of osteoarthritic knee pain: results from a prospective, multicenter, randomized, cross-over trial. Reg. Anesth. Pain Med. 44(4), 499-506 (2019).

- This is a clinical trial demonstrating the efficacy of CRFA compared with steroid injections in the management of knee OA pain. The majority of subjects receiving CRFA receive pain relief lasting at least 12 months.

10. Tran J, Peng PWH, Lam K, Baig E, Agur AMR, Gofeld M. Anatomical study of the innervation of anterior knee joint capsule: implication for image-guided intervention. Reg. Anesth. Pain Med. 43(4), 407-414 (2018).

- This is an anatomic study of the nerves innervating the knee joint capsule, which provides the roadmap for probe placement.

11. Fonkoué L, Behets C, Kouassi JÉK et al. Distribution of sensory nerves supplying the knee joint capsule and implications for genicular blockade and radiofrequency ablation: an anatomical study. Surg. Radiol. Anat. 41(12), 1461-1471 (2019).

12. Franco CD, Buvanendran A, Petersohn JD, Menzies RD, Menzies LP. Innervation of the anterior capsule of the human knee: implications for radiofrequency ablation. Reg. Anesth. Pain Med. 40(4), 363-368 (2015).

13. Mettler FA, Huda W, Yoshizumi TT, Manesh M. Effective doses in radiology and diagnostic nuclear medicine: a catalog. Radiology 248(1), 254-263 (2008).

14. US FDA. Initiative to reduce unnecessary radiation exposure from medical imaging. https:/www.fda.gov/radiation-emitting-products/initiative-reduce-unnecessary-radiation-exposure-medical-imaging/white-p aper-initiative-reduce-unnecessary-radiation-exposure-medical-imaging

15. Kim DH, Lee MS, Lee S, Yoon SH, Shin JW, Choi SS. A prospective randomized comparison of the efficacy of ultrasound- vs fluoroscopy-guided genicular nerve block for chronic knee osteoarthritis. Pain Physician 22, 139-146 (2019). 